\title{
Present status of Rare-RI Ring project in RIKEN RI Beam Factory
}

\author{
Yoshitaka Yamaguchi* \\ RIKEN Nishina Center \\ E-mail: yamagucheribf.riken.jp
}

M. Wakasugi, H. Suzuki, T. Fujinawa, T. Uesaka, and Y. Yano

RIKEN Nishina Center

\section{A. Ozawa, D. Nagae}

Institute of Physics, University of tsukuba

\section{T. Yamaguchi, T. Suzuki}

Department of Physics, Saitama University

\section{T. Kikuchi, W. Jiang, T. Sasaki}

Department of Electrical Engineering, Nagaoka University of Technology

\section{A. Tokuchi}

Pulsed Power Japan Laboratory Ltd.

\begin{abstract}
In the near future, short-lived and rare nuclei will be generated with a production rate of about one particle per day by uranium in-flight fission with an intensity of 1 particle micro-Amp at the accelerator complex in RIBF. Our apparatus, named "Rare-RI Ring", will be constructed at the downstream of an in-flight RI-beam separator BigRIPS. It consists of three main parts: an injection line, a fast response kicker system, and a cyclotron-like storage ring. The combination of an injection line and a fast response kicker system enables us to inject short-lived rare nuclei into the isochronous ring one by one. The isochronous ring, which consists of six magnetic sectors and straight sections, allows us to determine the mass with an order of $10^{-6}$ precision for even only one particle, by measuring the revolution time of the particle under the condition of precise isochronism. Here, we report the present status of this project.
\end{abstract}

8th International Conference on Nuclear Physics at Storage Rings-Stori11,

October 9-14, 2011

Laboratori Nazionali di Frascati, Italy

\footnotetext{
* Speaker.
} 


\section{Principles of mass measurement}

Nuclear masses are one of the most important quantities for understanding nuclear properties. Systematic mass measurements, especially for neutron-rich exotic nuclei that locate very far from the stability, are essential for solving the rapid neutron-capture process ( $r$-process) path. However, the nuclei in such regions are much short-lived and have a very low production rate even with the powerful accelerator complex in RIBF [1, 2, 3], an ingenious apparatus is needed. We propose a unique apparatus to measure the masses of short-lived rare nuclei with an order of $10^{-6}$ precision.

According to the cyclotron principle, revolution time is the inverse of cyclotron frequency. In addition, when an isochronous optics is achieved, the revolution time $\left(T_{0}\right)$ is expressed as

$$
T_{0}=2 \pi \frac{m_{0}}{q} \frac{1}{B} \gamma_{0}=2 \pi \frac{m_{0}}{q} \frac{1}{B_{0}}
$$

where $B_{0}$ is a uniform magnetic field, and $\gamma_{0}$ is the relativistic factor defined as $\gamma_{0}=1 / \sqrt{\left(1-\beta_{0}^{2}\right)}$ where $\beta_{0}=v / c$. The isochronous magnetic field at specific momentum spaces inside the ring is calibrated with $10^{-6}$ or higher accuracy using an already known stable nucleus. In such an isochronous ring, we measure the revolution time of an unknown mass nucleus, which has almost the same $m / q$ value as the stable nucleus. In such nuclei with slightly different mass-to-charge ratios, i.e., $m_{1} / q=m_{0} / q+\Delta\left(m_{0} / q\right)$, isochronism is no longer fulfilled. In this case, $m_{1} / q$ can be expressed as

$$
\frac{m_{1}}{q}=\frac{m_{0}}{q} \frac{T_{1}}{T_{0}} \frac{\gamma_{0}}{\gamma_{1}}=\frac{m_{0}}{q} \frac{T_{1}}{T_{0}} \sqrt{\frac{1-\beta_{1}^{2}}{1-\left(\frac{T_{1}}{T_{0}} \beta_{1}\right)^{2}}},
$$

where $T_{1}$ is the revolution time for nuclei with $m_{1} / q$ and $\gamma_{1}=1 / \sqrt{1-\beta_{1}^{2}}$. To evaluate the mass of nuclei that are not isochronous, correction for velocity $\beta_{1}$ is required. The relative differential value of $m_{1} / q$ is expressed as

$$
\frac{\delta\left(m_{1} / q\right)}{m_{1} / q}=\frac{\delta\left(m_{0} / q\right)}{m_{0} / q}+\frac{\delta\left(T_{1} / T_{0}\right)}{T_{1} / T_{0}}+k \frac{\delta \beta_{1}}{\beta_{1}}
$$

where

$$
k=-\frac{\beta_{1}^{2}}{1-\beta_{1}^{2}}+\left(\frac{T_{1}}{T_{0}}\right)^{2} \frac{\beta_{1}^{2}}{1-\left(T_{1} / T_{0}\right)^{2} \beta_{1}^{2}} .
$$

The measurement is based on the above equations. When we require an accuracy in the order of $10^{-6}$ for $1 \%$ difference in mass, we must measure $T_{1}$ with $10^{-6}$ accuracy and $\beta_{1}$ with $10^{-4}$ accuracy.

Figure 1 shows the conceptual design for our apparatus. When a produced secondary particle passes through the timing detector at F3 of the BigRIPS 㺻, a trigger pulse is generated. The trigger pulse is transmitted to a kicker system via a high frequency coaxial transmission cable. The kicker magnet is then immediately excited by a thyratron switch. In the meanwhile, the particle goes through the BigRIPS, SHARAQ [5, 6] and an injection line and is injected into an equilibrium orbit of the isochronous ring using septum and kicker magnets. After the particle revolves about 2000 times on the equilibrium orbit, it is extracted from the isochronous ring using another septum and the same kicker magnets. The revolution time of the particle is measured with an accuracy of better 


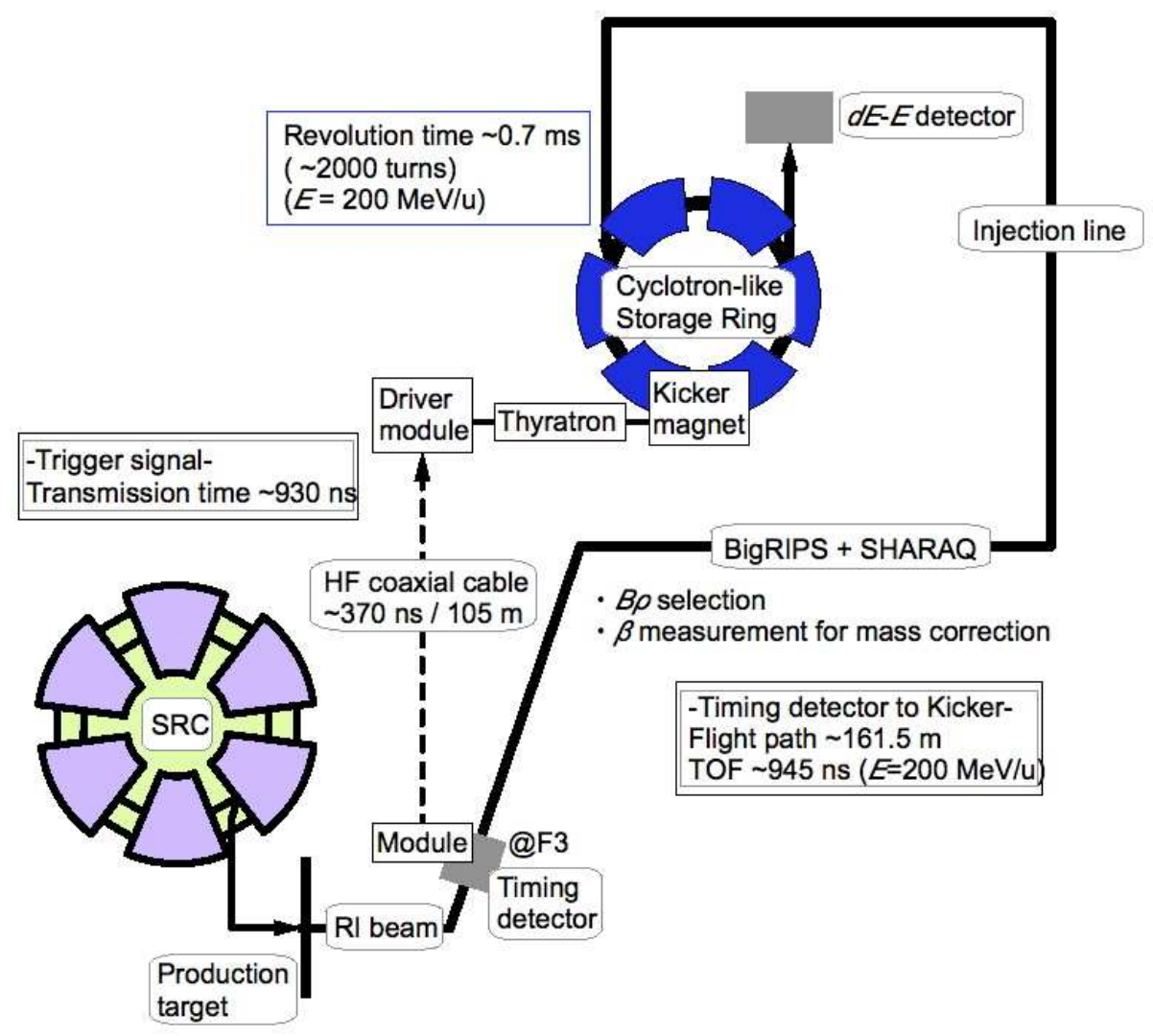

Figure 1: Conceptual design for our apparatus.

than $10^{-6}$ under the condition of precise isochronism. This concept enables mass measurement of even one particle. In addition, it is suitable to measure the mass for $r$-process region, since the measurement time is short enough $(<1 \mathrm{~ms})$.

We refer to our injection method as the individual injection method hereafter. For individual injection, it is necessary to excite the kicker magnet before the particle arrives at the kicker. The distance between F3 and the kicker is about $161.5 \mathrm{~m}$. Therefore, the flight time of a particle with an energy of $200 \mathrm{MeV} /$ nucleon is about $945 \mathrm{~ns}$, whereas the time taken for kicker excitation should be less than $945 \mathrm{~ns}$. The response time of the kicker power supply plays a key role in shortening the time taken for kicker excitation. Recently, we have succeeded in shortening the response time by the required amount. A feasibility study was performed by using a model kicker system [7] and the details of which are described in section 2.

To achieve precise isochronism inside the ring, we adopt a technique such as trim-coils used in the cyclotron. A design study is performed by using our simulation program [8]; the details of this design are described in section 3.

\section{Fast response kicker system}

The model kicker system consists of a driver module, a CX1171 thyratron, a distributed type 
kicker magnet, and a termination resistor. Figure 2 shows a block diagram of the model kicker system. Charge and discharge trigger pulses are generated using a pulse generator during the

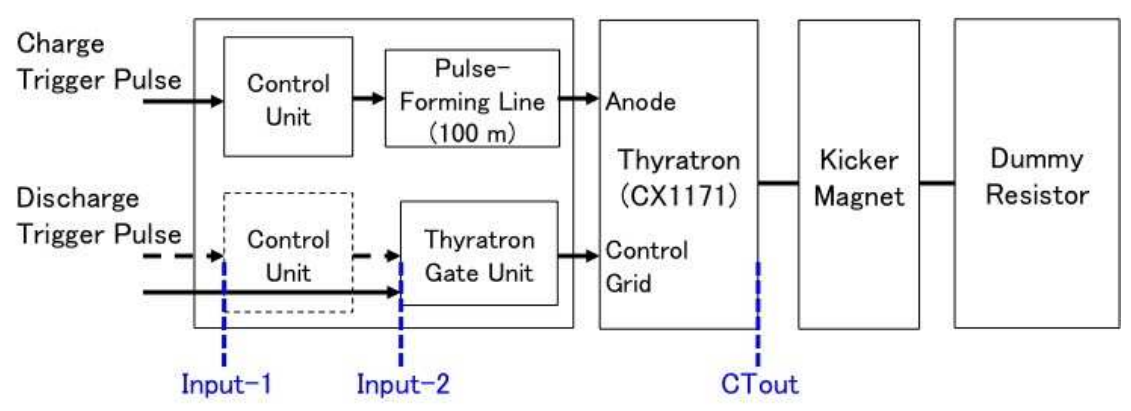

Figure 2: Block diagram of model kicker system.

measurements. A control unit adjusts the timing of the pulses. The duration of the flat-top is fixed by the length of the pulse-forming line. An appropriate grid pulse for a thyratron is formed in the thyratron gate unit. The thyratron is a high-peak-power electrical switch. It consists of a cathode, an anode, a ground grid, a control grid, an auxiliary grid, a cathode heater, and a reservoir heater. CTout denotes the current transformer output of the thyratron.

We first examined the initial performance of the model kicker system. The response time (between Input-1 and CTout) is about $2.67 \mu$ s when the charging voltage is $20 \mathrm{kV}$ and the reservoirheater voltage is $5.3 \mathrm{~V}$. Here, the measurement timing is defined as $10 \%$ of the full strength of each signal. In our method, the control unit for discharge is not necessary for injection. Therefore, we can send the discharge trigger pulse directly to the thyratron gate unit. The response time (between Input-2 and CTout) is $550 \mathrm{~ns}$ when the charging voltage is $20 \mathrm{kV}$ and the reservoir-heater voltage is $5.3 \mathrm{~V}$. However, this response time does not satisfy our requirement.

We constructed a new thyratron gate board to replace the original thyratron gate unit. Figure 3 shows the block diagram of the model kicker system with the new thyratron gate board, as well as a photograph of this board. The new thyratron gate board consists of four MOSFETs and four pulse transformers (PTs). To shorten the rise time of the PTs, four PTs are used in parallel on the primary side and in series on the secondary side. The thyratron housing was connected directly to the dummy resistor to concentrate the measurement of the response time. For the model kicker system shown in Fig. 3, we achieved a response time of about 275 ns between Input-3 and CTout for the same values of charging and reservoir-heater voltages as mentioned before. The pulse takes about $30 \mathrm{~ns}$ to cross the optical-link part. A time period of about $75 \mathrm{~ns}$ is required to form an appropriate grid pulse. The driving time of the thyratron is about 170 ns. Figure 4 shows the response time between Input-3 and CTout as a function of the charging voltage. The response time depends on the charging voltage and stabilizes when the charging voltage is $20 \mathrm{kV}$ or more.

Consequently, the time taken for kicker excitation is expected to be about 930 ns. It takes about 55 ns to generate the trigger pulse at F3. The pulse takes about 370 ns to transmit through the high frequency cable. The achieved response time of the kicker power supply is about $275 \mathrm{~ns}$. The delay time between CTout and the flat-top center of kicker magnetic field is estimated to be about $230 \mathrm{~ns}$. 


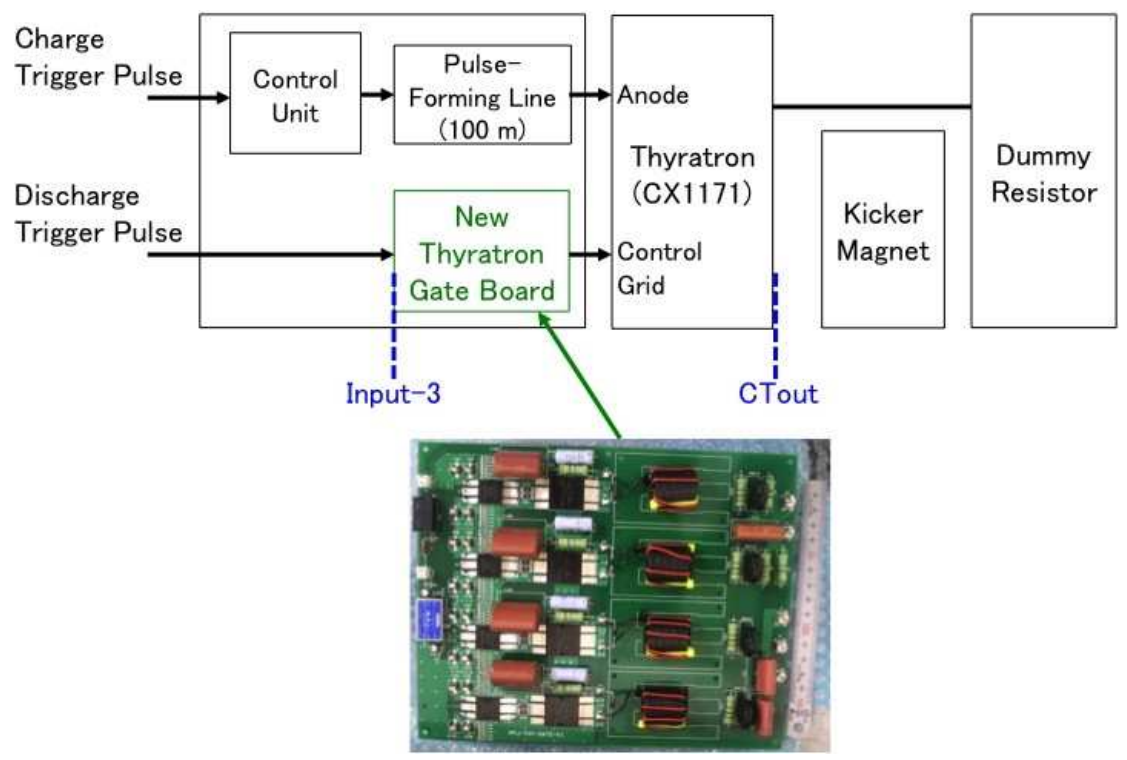

Figure 3: Block diagram of model kicker system with new thyratron gate board and photograph of this board.

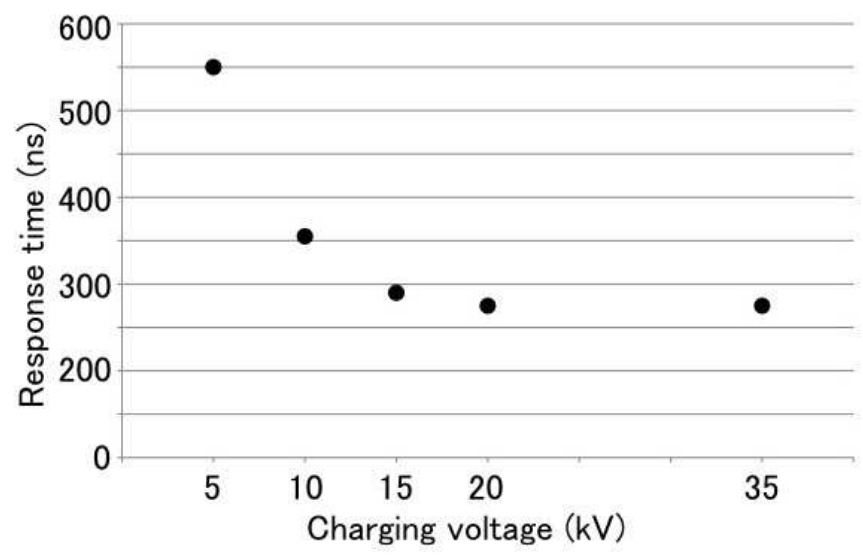

Figure 4: Response time between Input-3 and CTout as function of charging voltage.

Our kicker system will be used not only for injection but also for extraction. The measurement time is estimated to be about $0.7 \mathrm{~ms}$, so the kicker magnet must be excited twice within $0.7 \mathrm{~ms}$. It is necessary to perform the charge twice within $0.2 \mathrm{~ms}$, since the recovery time of the CX1171 thyratron is about $0.5 \mathrm{~ms}$. A high-speed repetitive charging system is necessary. We have begun to examine the feasibility by using the model kicker system.

\section{Isochronous design}

Figure 5 shows the arrangement plan for our apparatus. The floor level is the same as that of the SHARAQ spectrometer [5]. The injection line, which starts from the end of the final focal plane 


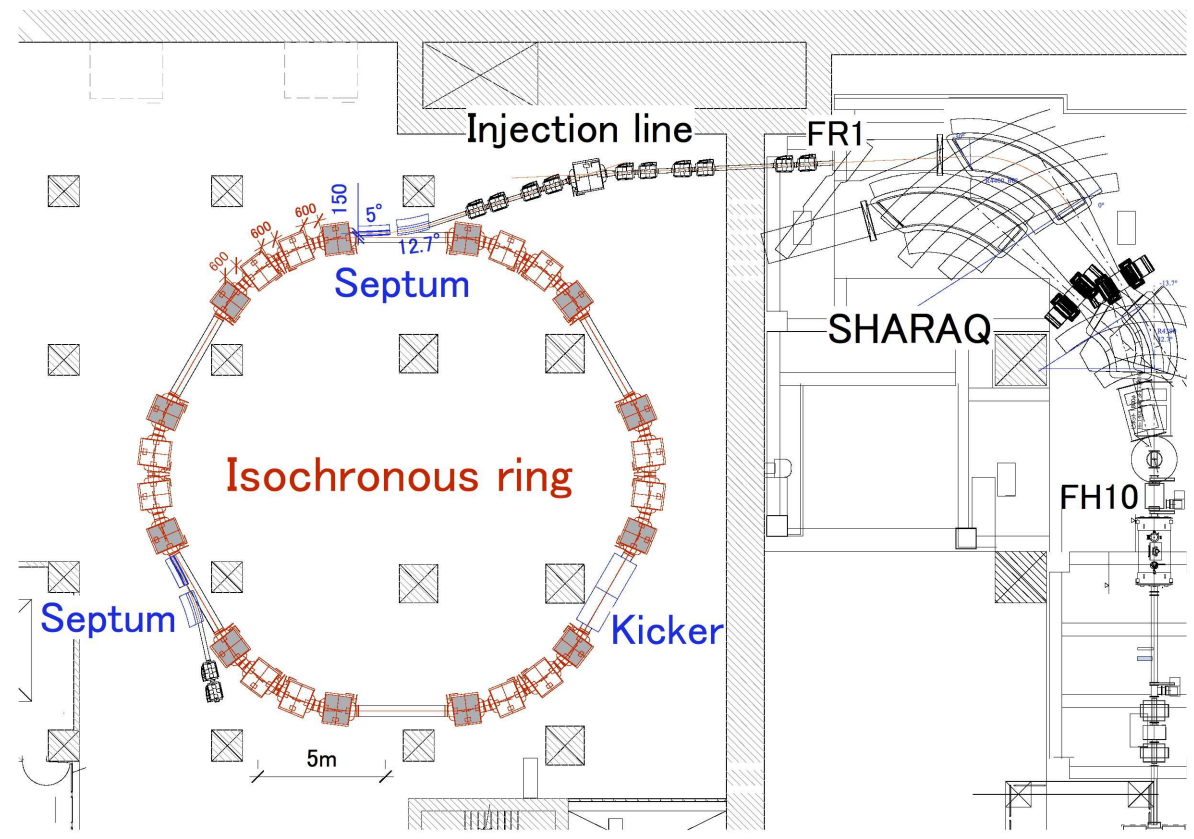

Figure 5: Arrangement plan for our apparatus.

of SHARAQ, is much shorter than that in the previous design [9]. It consists of ten quadrupole magnets, which were obtained from the KEK Proton Synchrotron (KEK-PS), and one dipole magnet with a $15^{\circ}$ bend. This dipole magnet was obtained from the cooler synchrotron TARNII. We adopt single-turn injection by using septum and kicker magnets. The phase advance from the septum end to the kicker center is about $3 \pi / 2$. The septum, which is operated as a DC magnet, is divided into two magnets to reduce the load exerted on it. The bending angles of the two septum magnets are $12.7^{\circ}$ and $5^{\circ}$, respectively. After passing through the septum magnets, a particle is injected into the isochronous ring from outside. The injected particle is transported to the kicker magnet, and it is then kicked into the equilibrium orbit with dispersion and emittance matching. The isochronous ring consists of six magnetic sectors, and each magnetic sector consists of the four dipole magnets of TARNII. The TARNII Dipole (TARNII-D) is a rectangular bending magnet with a radially homogeneous magnetic field; however, it is not acceptable in our isochronous design. To create an appropriate isochronous magnetic field, we place a thin laminated iron plate on the pole face of TARNII-D as the first step. Furthermore, we install trim-coils in the TARNII-D to tune the isochronous magnetic field precisely. This remodeling, which is optimized to $200 \mathrm{MeV} /$ nucleon, will be performed for the two outer dipoles among the four dipoles in each magnetic sector. To evaluate the isochronism inside the ring in detail, we use our simulation program [8]. We track particles in the magnetic sector by assuming that the particles revolve in circular orbits within a small spatial segment. The magnetic sector is divided into 210 sub-sectors, and the calculated revolution time of particle. The magnetic field in each segment is deduced from the results of a three-dimensional field analysis calculation program.

The left-hand side of Fig. 6 shows a model of TARNII-D with a thin laminated iron plate. The 

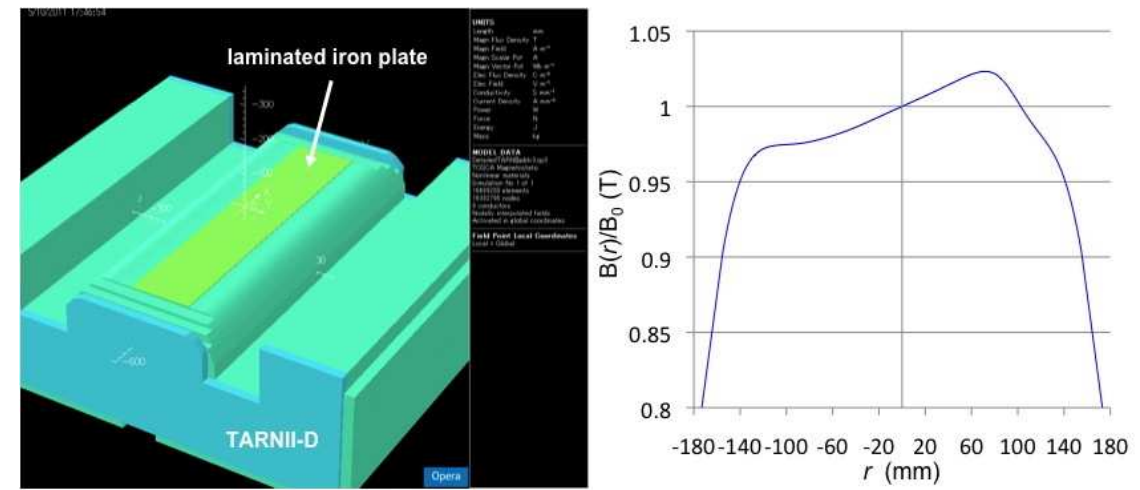

Figure 6: Model of TARNII-D with thin laminated iron plate is shown on the left side. Radial distribution of magnetic field strength with the effect of the thin laminated iron plate is shown on the right side.

radial distribution of the magnetic field strength is tilted, as shown on the right-hand side of Fig. 6 by laminating the thin iron plates in the shape of stairs. Figure 7 shows a simulation result that expresses the relative difference of revolution times (2000 turns) as a function of momentum. It was found that the placement of the thin laminated iron plate is optimized at $1.5 \mathrm{Tesla}$, as indicated by the solid circles. In this measurement technique, the energy of the particle is fixed. Therefore, it is necessary to change the magnetic field strength of the ring each time we measure the mass of particles with different $\mathrm{m} / \mathrm{q}$. The result indicated by red circles assumes $1.0 \mathrm{Tesla}$, and this magnetic field strength corresponds to a particle of $\mathrm{m} / \mathrm{q}=2$. The result indicated by blue circles assumes 1.6 Tesla, and this magnetic field strength corresponds to a particle of $\mathrm{m} / \mathrm{q}=3$. Consequently, the isochronism for $1 \%$ momentum spaces inside the ring is achieved to be less than $10^{-4}$ using the thin laminated iron plate.

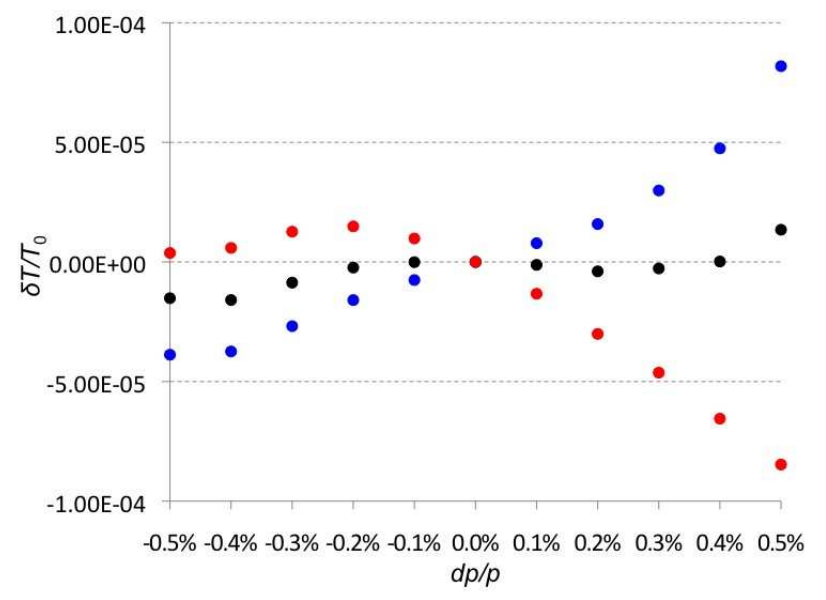

Figure 7: Simulation results expressing the relative difference of revolution times (2000 turns) as a function of momentum. Results indicated by black circles assume 1.5 Tesla, red circles assume 1.0 Tesla, and blue circles assume 1.6 Tesla. 
For more fine tuning, we use trim-coils. The simulation results under ideal condition show that the isochronism for $1 \%$ momentum spaces inside the ring achieved is less than $10^{-6}$ if we place the trim-coils properly.

\section{Summary}

We developed a fast response kicker system. Because the response time of the kicker power supply becomes fast, we will be able to use the self-trigger individual injection method with an energy of $200 \mathrm{MeV} /$ nucleon. We chose the ring design that ensures isochronism of $10^{-6}$ at specific momentum spaces. The shape of the thin laminated iron plate was selected. The remaining task is to fix the placement of the trim-coils. Design studies for other devices, such as the septum magnets, schottky pick-up, and RF cavity, are still in progress.

\section{References}

[1] Y. Yano, The RIKEN RI Beam Factory Project: A status report, Nucl. Instrum. and Methods $\mathbf{B 2 6 1}$ (2007) 1009.

[2] T. Ohnishi, et al., Identification of New Isotopes ${ }^{125} \mathrm{Pd}$ and ${ }^{126} \mathrm{Pd}$ Produced by In-Flight Fission of 345 MeV/nucleon ${ }^{238}$ U: First Results from the RIKEN RI Beam Factory, J. Phys. Soc. Jpn. 77 (2008) 083201.

[3] T. Ohnishi, et al., Identification of 45 New Neutron-Rich Isotopes Produced by In-Flight Fission of a ${ }^{238}$ U Beam at 345 MeV/nucleon, J. Phys. Soc. Jpn. 79 (2010) 073201.

[4] T. Kubo, In-flight RI beam separator BigRIPS at RIKEN and elsewhere in Japan, Nucl. Instrum. and Methods B204 (2003) 97.

[5] T. Uesaka, et al., The high resolution SHARAQ spectrometer, Nucl. Instrum. and Methods $\mathbf{B 2 6 6}$ (2008) 4218.

[6] T. Kawabata, et al., High resolution beam line for the SHARAQ spectrometer, Nucl. Instrum. and Methods B266 (2008) 4201.

[7] T. Ohkawa, et al., Development of the kicker magnet for muses, Nucl. Instrum. and Methods $\mathbf{A 5 4 7}$ (2005) 287.

[8] I. Arai, et al., Beam optics simulation of Rare-RI Ring at RI Beam Factory in RIKEN, in proceedings of STORI'08 conference (2008) 498.

[9] Y. Yamaguchi, et al., Rare-RI ring project at RIKEN RI beam factory, Nucl. Instrum. and Methods B266 (2008) 4575. 